\title{
A Numerical Approach for Hybrid Simulation of Power System Dynamics Considering Extreme Icing Events
}

\author{
Chen, Lizheng; Zhang, Hengxu; Wu, Qiuwei; Terzija, Vladimir
}

Published in:

IEEE Transactions on Smart Grid

Link to article, DOI:

10.1109/TSG.2017.2679109

Publication date:

2018

Document Version

Peer reviewed version

Link back to DTU Orbit

Citation (APA):

Chen, L., Zhang, H., Wu, Q., \& Terzija, V. (2018). A Numerical Approach for Hybrid Simulation of Power System Dynamics Considering Extreme Icing Events. IEEE Transactions on Smart Grid, 9(5), 5038-5046.

https://doi.org/10.1109/TSG.2017.2679109

\section{General rights}

Copyright and moral rights for the publications made accessible in the public portal are retained by the authors and/or other copyright owners and it is a condition of accessing publications that users recognise and abide by the legal requirements associated with these rights.

- Users may download and print one copy of any publication from the public portal for the purpose of private study or research.

- You may not further distribute the material or use it for any profit-making activity or commercial gain

- You may freely distribute the URL identifying the publication in the public portal 


\title{
A Numerical Approach for Hybrid Simulation of Power System Dynamics Considering Extreme Icing Events
}

\author{
Lizheng Chen, Student Member, IEEE, Hengxu Zhang, Member, IEEE, Qiuwei Wu, Senior Member, \\ IEEE, Vladimir Terzija, Fellow, IEEE
}

\begin{abstract}
The global climate change leads to more extreme meteorological conditions such as icing weather, which have caused great losses to power systems. Comprehensive simulation tools are required to enhance the capability of power system risk assessment under extreme weather conditions. A hybrid numerical simulation scheme integrating icing weather events with power system dynamics is proposed to extend power system numerical simulation. A technique is developed to efficiently simulate the interaction of slow dynamics of weather events and fast dynamics of power systems. An extended package for PSS/E enabling hybrid simulation of icing event and power system disturbance is developed, based on which a hybrid simulation platform is established. Numerical studies show that the functionality of power system simulation is greatly extended by taking into account the icing weather events.
\end{abstract}

Index Terms-- extreme meteorological conditions, hybrid simulation, icing weather, power system modeling

\section{INTRODUCTION}

$\mathrm{M}$ OST of overhead transmission lines and towers are exposed to weather events. It is well known that weather conditions significantly affect the failure rates of transmission lines [1], [2]. The climate change and global warming have caused increasing economic losses from extreme weather events in the scale not experienced in the past. On Aug. 29th, 2015, a fierce wind storm swept through southern British Columbia, toppling trees, knocking down power lines and leaving about 500,000 BC Hydro customers without power supply overnight [3]. Data from the North American Electric Reliability Corporation (NERC) show that more than $80 \%$ of all outages in the period 2003-2012 were weather-related, and almost $18 \%$ of the outages were cold weather-related [4]. The ice storm is one of the typical adverse weather events that severely threaten the operation of transmission lines [5]. Ice accretions on power transmission lines may result in various damages to power system infrastructure, e.g. transmission line breaking, tower falling,

This work was jointly supported by National Natural Science Foundation of China (No:51477092) and the State Grid Corporation of China.

Lizheng Chen and Hengxu Zhang are both with the Key Laboratory of Power System Intelligent Dispatch and Control of the Ministry of Education (Shandong University), Jinan 250061, China (e-mail: chenlizheng120@ 163.com; zhanghx@sdu.edu.cn).

Qiuwei Wu is with the Department of Electrical Engineering, Technical University of Denmark, Kgs. Lyngby, DK 2800 (e-mail:qw@elektro.dtu.dk).

Vladimir Terzija is with the School of Electrical and Electronic Engineering, University of Manchester and the Key Laboratory of Power System Intelligent Dispatch and Control of the Ministry of Education (Shandong University), Jinan 250061, China (e-mail: vladimir.terzija@manchester.ac.uk) and insulator flashover [6]. Evaluating the impacts of extreme icing weather events on power systems is an important issue related to power system security and risk assessment [7], [8]. Smart grid development also pushes forward requirements of power systems to survive extreme weather conditions [9], [10].

Research on extreme icing weather events has been conducted for a long period. To collect data and evaluate the effects of icing weather events, a lot of work has been done on ice loading detection [11], ice accretion modeling [12], [13], numerical simulation techniques [14], statistical analysis [15] and ice melting approaches [16]. In [11], a capacitive measurement of detecting the ice formation and accumulation on an overhead transmission line was used to identify the factors that affect ice accretion. Various models were proposed to describe the dynamic process of ice accretion, e.g. models of Lenhard, Goodwin, and Makkonen. In [14], the numerical simulation method for icing weather events, i.e. line galloping amplitude of iced transmission lines after ice shedding, was investigated. Statistical analysis with historical data illustrated the pattern of ice loading on transmission lines, failure of towers, and lines tripping [15]. To prevent ice accretion, relevant models for glaze ice accretion and de-icing were discussed in [16]. The de-icing strategies by channeling load current were developed for transmission networks in [17].

Power system simulation has been used for a long time in the electrical industry. Initially, only power grid models were considered in the simulation, which modeled various components to investigate the detailed electromagnetic and electromechanical transients. Then economic factors were added to the simulation models to meet the need of economical operation of power systems. However, the meteorological system simulation is usually separated from the electromechanical dynamic simulation though the two dynamic processes happen simultaneously and interact with each other. More frequent occurrence of extreme weather events and more serious damages caused by them drive the need of extending the functionality of power system simulation. Therefore, a hybrid simulation scheme combining meteorological dynamics and power system dynamics is of great importance to improve the simulation framework of power systems [18].

How to integrate the two systems into a coherent simulation framework is challenging work as great differences exist in meteorological dynamics and power system dynamics. For the icing weather event simulation, the ice accretion modeling and analysis of the force on transmission lines and towers are important. For the electromechanical event 
simulation, the key problem is how to transfer weather events into electrical disturbances. When the two processes are simulated in a united framework, more problems have to be solved. Firstly, the two processes interact with each other. Icing weather events may cause flashover or transmission line tripping and affect power flow of the power grid. Current of a transmission line changes the rate of ice accretion on it. Secondly, time scales of the two processes are different. Several hours, even days, may be possible for the meteorological process. However, for the electrical process, especially the electromechanical process, only a few seconds are needed to be simulated in detail. Finally, the efficiency of a long time duration simulation of meteorological dynamics and power system dynamics is very important to satisfy the requirements of a large scale power system.

Until now, no integrated framework has been developed for simulating and assessing power system dynamics with extreme weather events in detail. How to integrate weather event dynamics with power system dynamics to extend the functions of the traditional power system numerical simulation is challenging work. The main contribution of this manuscript is developing the hybrid simulation scheme integrating icing events with power system dynamics, and the technique to carry out the long time duration hybrid simulation with high efficiency. With the hybrid simulation method, the detailed dynamic process of power systems and meteorological systems, and the interaction of them can be revealed. The hybrid simulation method helps develop the comprehensive risk assessment of power systems under extreme weather conditions.

The paper is organized as follows: Section II presents the framework of the proposed hybrid numerical simulation of power systems considering icing weather events. Section III describes the implementation of the hybrid simulation framework. In Section IV, case studies are presented to verify the proposed method, followed by conclusions.

\section{Hybrid Simulation FrameWORK FOR POWER SySTEMS CONSIDERING EXTREME ICING EVENTS}

\section{A. Hybrid Simulation Model and Framework}

For the power system consisting of various components (like transmission lines, machines, or loads), part of state variable values are known. When the system is in a steady state, key state variables can be obtained through power flow calculation expressed as algebraic equations. When electrical faults occur, dynamic trajectories of variables changed with time can be calculated through power system transient simulation expressed as differential equations. These two situations can be formulated as,

$$
\left\{\begin{array}{l}
\dot{\mathbf{x}}=F_{e}(\mathbf{x}, \mathbf{y}) \\
0=G_{e}(\mathbf{x}, \mathbf{y})
\end{array}\right.
$$

where

$\mathbf{x}$ Vector of state variables of power system (rotor angle, speed and internal transient voltages,...);

$\mathbf{y}$ Vector of algebraic variables of power system (time, impedance,...);

$\mathbf{F}_{\mathbf{e}} \quad$ Vector of differential equations which describe electromechanical transient process;

$\mathbf{G}_{\mathbf{e}}$ Vector of algebraic equations which provide constraint of power flow.

In the meteorological system of ice accretion, the values of state variables (weight or rate of ice accretion) can be deduced with algebraic variables (temperature, wind speed, precipitation,...) through the ice accretion model with the mathematical function expression as (2).

$$
\left\{\begin{array}{c}
\dot{\mathbf{z}}=\mathbf{F}_{m}(\mathbf{z}, \mathbf{h}) \\
\mathbf{0}=\mathbf{G}_{m}(\mathbf{z}, \mathbf{h})
\end{array}\right.
$$

where

$\mathbf{z}$ Vector of state variables of the meteorological system (weight or rate of ice accretion);

h Vector of algebraic variables of the meteorological system (temperature, precipitation,...);

$\mathbf{F}_{\mathbf{m}}$ Vector of differential equations which describe meteorological dynamic process;

$\mathbf{G}_{\mathbf{m}} \quad$ Vector of algebraic equations of meteorological dynamic process.

As mentioned above, the two systems interact with each other by current and other variables. For example, when the current of transmission lines changes with load fluctuation, the heating effect of lines will lead to the change of the ice accretion rate $\left(R_{I}, R_{I} \in \mathbf{z}\right)$. When a meteorological event occurs and results in line fault, the topology of power grid will be changed and relevant electrical responses will be triggered. On the other hand, when the weight of ice accretion ( $M_{i}, M_{i} \in \mathbf{z}$ ) reaches the force limit and results in line breaking, the current of transmission lines will be redistributed.

To reflect the dynamic relationship between the meteorological system and power system, a hybrid simulation framework is established as shown in Fig. 1. Meteorological data and electrical information are input information of the hybrid simulation module which combines icing events simulation and power system simulation together and output ice accretion value and electrical responses for further analysis.

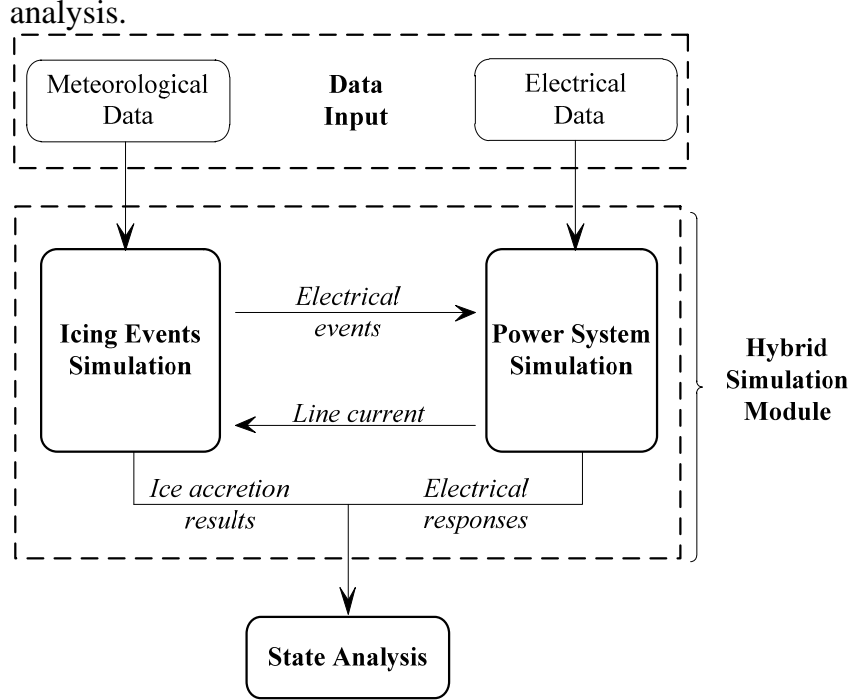

Fig.1 Hybrid simulation framework of meteorological and power systems.

In hybrid simulation module, two main functions are 
included. Firstly, meteorological events affect electrical process through the topology change. For meteorological events simulation, the ice accretion model is constructed to simulate the process of ice accretion. With the input meteorological information, weight of ice accretion on transmission lines can be calculated and be exported for mechanical accidents assessment. If the growth of ice accretion results in mechanical accidents, the relevant electrical disturbances will be triggered, and power system states (e.g. current of transmission lines) will be changed. Secondly, the change of power system states can also react on the meteorological system. The heat produced by current of transmission lines will also influence the process of ice accretion. The schematic diagram of the interface of hybrid simulation system is shown in Fig. 2.

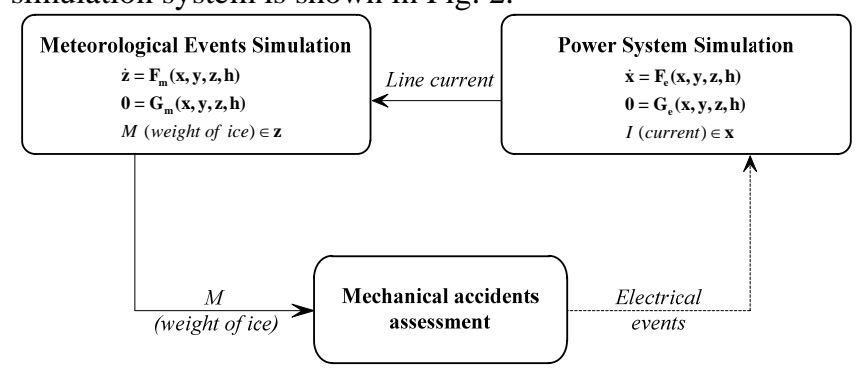

Fig.2 Interface of the hybrid simulation scheme.

The interface connects meteorological events with power system dynamics and transforms the results of the former into the inputs of the latter, as shown in Fig. 3. Under icing weather conditions, transmission lines breaking, tower falling and isolator ice flashover may happen and threaten power grid secure operation. In this paper, only the transmission line breaking is modeled in detail in the proposed hybrid simulation framework.

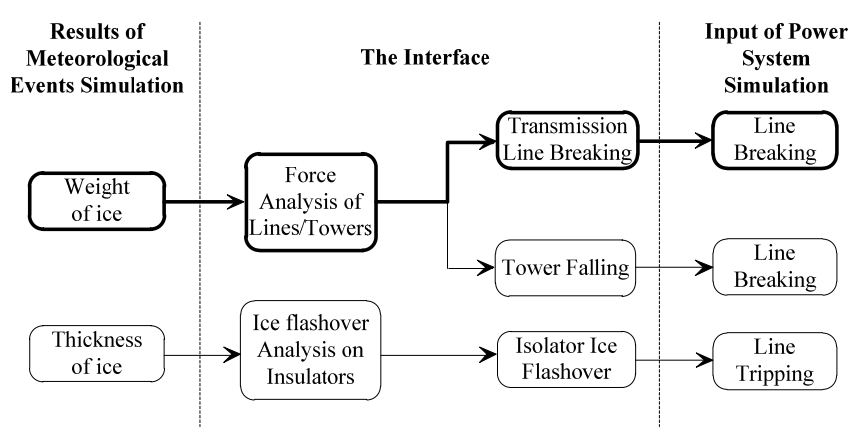

Fig.3 Relationship between meteorological events and power system disturbances.

\section{B. Time Scale Differences and Combination}

The hybrid simulation framework consists of two main simulation modules which have great difference in time scale. For the meteorological system, extreme weather events may last for several hours, even for several days. However, for power systems, especially for electromechanical dynamic simulation, the dynamic process of only ten or twenty seconds after disturbances is of interest. Therefore, a scheme is proposed as shown in Fig. 4.

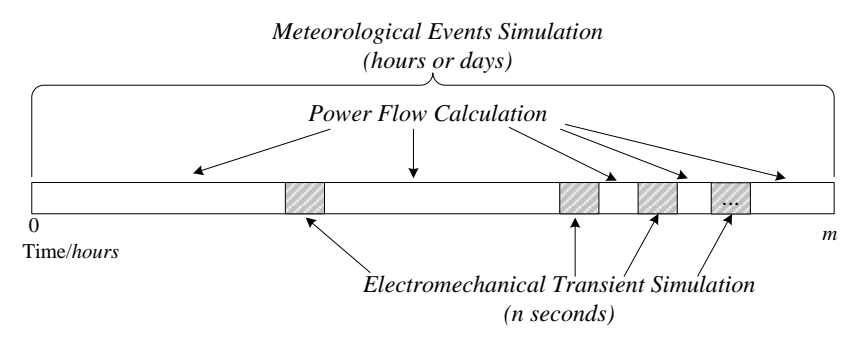

Fig.4 Simulation time of various simulation processes.

Assume the whole simulation period as $m$ hours, during which meteorological simulation is performed all the time period. With the growth of the ice load weight, relevant mechanical events may occur, like transmission line breaking or tower falling. After these mechanical events are converted to relevant electrical disturbances, the power system electromechanical dynamics simulation will be conducted for $n$ seconds. To improve simulation efficiency, the process of power system operation in the interval period of two adjacent faults is hourly 'freezed', without any fluctuation of loads during one hour, but updating the load once per hour. When the system goes into a steady state (usually twenty seconds after a disturbance), power flow calculations are performed to obtain the steady state, instead of performing transient simulation all the time. It is a technique to greatly improve simulation efficiency.

\section{Development of Hybrid Simulation Platform}

The ice accretion process and electrical dynamic hybrid simulation are the key issues to analyze the detailed evolving process of ice disasters. Such a hybrid simulation tool is not available in the literature. To develop such a software package, there are two approaches: (1) design and develop a brand-new hybrid simulation program; (2) further develop existing mature commercial software.

A brand-new developed simulation program can provide excellent structural framework. However, it requires excessive work. Among existing commercial software packages, PSS/E's flexibility and strong modeling capacity make it suitable for developing such a hybrid simulation system. It provides transient simulation and extended term simulation function, and can realize switching between them. Flexible customized modeling methods and more than 600 application program interface (API) functions are available, which allow users to use Python or FORTRAN to realize complicated user defined functions. With these APIs, users can control the main program conditionally, and exchange data with external program in order to realize hybrid simulation.

\section{IMPLEMENTATION OF THE HYBRID SIMULATION CONSIDERING EXTREME ICING EVENTS}

The diagram of the proposed hybrid simulation framework is shown in Fig. 5. The details of every module are discussed. 


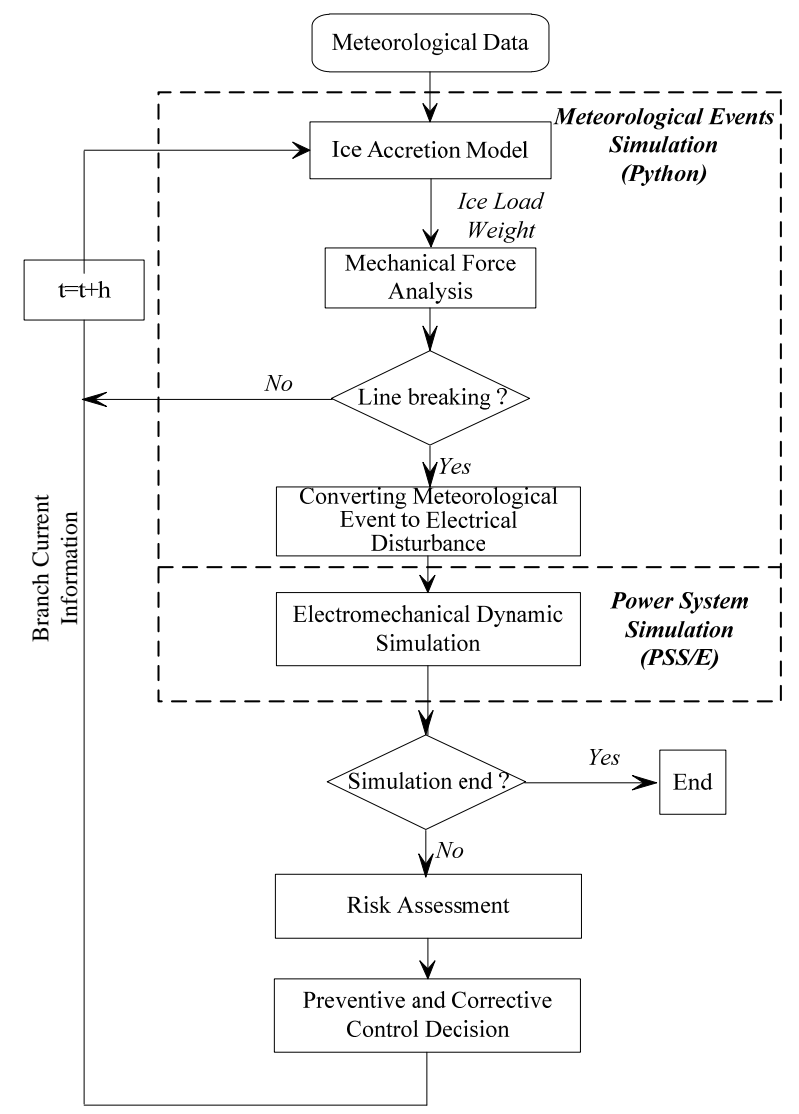

Fig.5 Hybrid simulation and control decision diagram.

\section{1) Meteorological Data}

Meteorological data of icing weather mainly refer to ambient weather conditions of transmission corridors. These conditions can be obtained from a meteorological information monitoring system or a numerical weather forecasting system. In this platform, the meteorological data are provided as input information, including temperature, precipitation, and wind speed.

In natural environment, meteorological condition differs with each other greatly, especially in a wide area. In the simulation case, identical weather condition along the same line is adopted based on two reasons: firstly, identical weather condition can simplify the simulation; secondly, in modern power grid, the monitoring of meteorological information is not sufficient enough. But, it does not mean the presented method is limited to the simplified weather condition. If detail weather condition along a transmission line is available, the presented hybrid simulation platform will allow dividing one transmission line into several sections, calculating the ice accretion of each section based on its corresponding weather condition, and then calculating the force on the transmission line according to the total ice weight of all the sections.

\section{2) Ice Accretion Model}

With the input meteorological data, ice accretion process can be simulated based on an appropriate model. Compared to establishing the hybrid simulation framework, the research on the ice accretion model is not our priority. Therefore, more attention is paid on the selection of models from existing work.

Up to now, several models have been developed to describe the process of ice accretion [19]-[22]. In [19], the ice accretion rate was mainly related to the ambient temperature. The model in [20] assumed the ice accretion rate as a result of the precipitation. In the third model, more factors were taken into consideration, however, the speed of raindrop was hard to be obtained. Ref. [22] considered the angle between cable orientation and wind besides temperature, wind speed, raindrop rate and transmission lines radius.

Previous work mainly focused on the detailed ice accretion process and shape prediction of ice loading on transmission lines. Actually the sum weight of ice and transmission lines is needed for further mechanical events analysis. And the heating effect of current has not been considered in those references above. So we consider the heating effect based on the model in [22] which can simulate the process of glaze ice accretion and output the sum weight of ice accretion and transmission lines.

The model adopted here is,

$$
M_{i}=\int_{0}^{\tau}\left[\frac{4}{\pi} r(t)\left(1+\frac{C_{a} V_{a}}{S_{w} r}\right)^{-1} S_{w} C_{w} C_{c} \sin \theta_{1} \sin \theta_{2}+C_{i} I\right] d t
$$

where

$M_{i} \quad$ Weight of ice accretion;

$r(t) \quad$ Radius of transmission lines with ice;

$C_{a} \quad$ Constant of ice accretion (1.64);

$V_{a} \quad$ Viscosity of air movement $\left(13.2 \times 10^{-6} \mathrm{~m}^{2} / \mathrm{s}\right)$;

$S_{w} \quad$ Speed of wind;

$r \quad$ Radius of transmission lines;

$C_{w} \quad$ Content of liquid water $\left(0.067 P^{0.846} \mathrm{~g} / \mathrm{m}^{3}\right)$;

$C_{c} \quad$ Collection coefficient $(=1$, when temperature is below $-2^{\circ} \mathrm{C}$ );

$\theta_{1} \quad$ Angle between raindrop and transmission lines $\left(60^{\circ}\right)$;

$\theta_{2} \quad$ Angle between wind and transmission lines ( $\left.45^{\circ}\right)$;

$C_{i} \quad$ Current heating effect coefficient (-0.1);

I Current of transmission lines.

In (3), the collision coefficient, freeze coefficient, collection coefficient and current heating effect are all considered. As shown in Fig. 6, the angle between the precipitation and transmission lines is set as $\theta_{1}$, and the angle between wind and transmission lines as $\theta_{2}$. Both the two angles are variable, but here we assume them as constant.

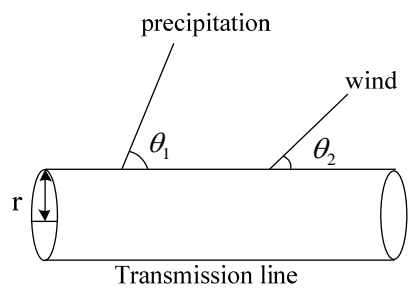

Fig.6 Angle of precipitation and wind with transmission line.

$r(t)$ is the radius of lines covered with ice and is equal to the sum of $\mathrm{r}$ and $\Delta r$.The radius of lines $r$ is assumed as $15 \mathrm{~mm}$ and $\Delta r$ is, 


$$
\Delta r=\frac{0.035}{1+\frac{21.65}{S_{w} D_{w}}}\left[R_{p}{ }^{2}+\left(0.2412 S_{w} R_{p}{ }^{0.846}\right)^{2}\right]^{1 / 2}
$$

where

$R_{p} \quad$ Rate of precipitation;

$D_{w} \quad$ Diameter of overcooled water droplets (10 $\left.\mu \mathrm{m}\right)$.

By the calculation of (3), the weight and rate of ice accretion can be obtained for further mechanical event analysis.

3) Mechanical Force Analysis

Too much ice loading on transmission lines may cause the line break force limit exceeded and result in line breaking. Ice covering also increases the probability of ice flashover, but it is not considered in the implementation of the current version platform to simplify the simulation.

Transmission line breaking is related to the stress, ice accretion thickness, ice accretion heterogeneity as well as wind speed and wind direction. In this paper, the trigger of transmission line breaking is assumed only related to the weight of ice load and the line force limit. When the total weight of a transmission line and ice covering exceeds the force limit, transmission line breaking will occur. The weight of ice on transmission lines can be obtained from the results of the ice accretion model.

Wind loading force modeling is another complex problem in this field, which is related to wind direction and ice shape. Wind can even lead to transmission line galloping. Wind loading force modeling issues are the future work to enhance the hybrid simulation scheme.

4) Line Breaking Assessment

This module converts total weight to the force on the transmission line, and compares it with the force limit. If the force applied on the transmission line exceeds its limit, line breaking will occur. Line breaking triggers electromechanical dynamic simulation of line trip disturbance, which is treated in the next module.

\section{5) Electromechanical Dynamic Simulation}

The electromechanical dynamic simulation triggered by disturbance caused by extreme ice loading is performed by PSS/E. The outputs of this module are the responses of the power system, usually only the variables of concern (i.e. generator angle, bus voltage, branch current, system frequency) are monitored by the user and exported for further analysis. In this hybrid simulation platform, the current of transmission lines is the most important variable that affects ice accretion, which is transferred to and updated in the ice accretion model. The other electrical variables will be analyzed for security and stability assessment until the end of simulation time.

\section{6) Risk Assessment and Control Decision}

Risk assessment module performs comprehensive assessment of the power system state under extreme weather conditions. The preventive and corrective control decision module aims to make decisions in different time horizons so as to prevent the system from undesired situations caused by icing events.

Unfortunately, the ice loading on transmission lines and total weigh of them can't be effectively controlled by preventive or corrective control. The progress of ice accretion depends mainly on weather conditions, slightly on line current.
On one hand, in order to melt ice covering on transmission lines, lager current is needed to produce more heat, and usually be used when the line is out of service. On the other hand, when the mechanical force limit is reached and the line is broken, lager load current means a more severe impact on the power grid. So the preventive or corrective controls here are to decrease the effects of line breaking on power system security.

\section{CASe STUdies}

\section{A. Case Descriptions}

\section{1) Grid Data}

Case studies were conducted using the 23-bus model provided by PSS/E, shown in Fig. 7. In the test system, 6 machines and 23 nodes are located in three areas.

Eight transmission lines are assumed to be exposed to the icing conditions. Transmission lines \#1 \#4 are located in AREA 1 , line \#5 and \#6 are located in AREA 2, and line \#7 and \#8 in AREA3.

Here, it is assumed that the force applied on a line, which is used to be compared with its force limit, is proportional to the total weight of the transmission line and ice load on it. Therefore, when the total weight exceeds the value deduced by the force limit, the transmission line will be tripped.

Transmission lines of different voltage levels can bear different force limits and have different spans, the detailed parameters are listed in TABLE I.

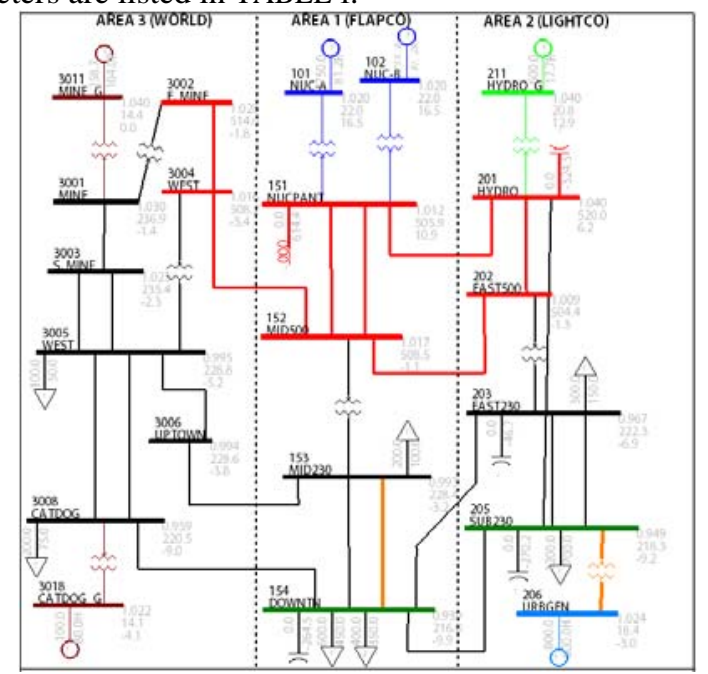

Fig.7 The power system with 23 nodes provided by PSS/E.

TABLE I

TRANSMISSION LINES INFORMATION

\begin{tabular}{cccccc}
\hline Number & $\begin{array}{c}\text { Voltage } \\
\text { Level/ } \\
\mathrm{kV}\end{array}$ & $\begin{array}{c}\text { Span/ } \\
\mathrm{km}\end{array}$ & $\begin{array}{c}\text { Weight/ } \\
(\mathrm{kg} / \mathrm{km})\end{array}$ & $\begin{array}{c}\text { Limit of } \\
\text { Tension Force/ } \\
\mathrm{kg}\end{array}$ & $\begin{array}{c}\text { Rating } \\
\text { Current/ } \\
\mathrm{A}\end{array}$ \\
\hline 1 & 500 & 1.2 & 1600 & 5000 & 1200 \\
2 & 500 & 1.1 & 1600 & 5000 & 1200 \\
3 & 500 & 0.9 & 1600 & 5000 & 1200 \\
4 & 500 & 0.8 & 1600 & 5000 & 1200 \\
5 & 220 & 1.2 & 960 & 3000 & 800 \\
6 & 220 & 1.1 & 960 & 3000 & 800 \\
7 & 220 & 0.9 & 960 & 3000 & 800 \\
8 & 220 & 0.8 & 960 & 3000 & 800 \\
\hline
\end{tabular}

2) Weather information 
The input weather information around transmission lines includes ambient temperature, precipitation and wind speed. The curves of temperature and precipitation changes are shown in Fig. 8. Temperature changing with time will reach minimum value $-10^{\circ} \mathrm{C}$ at $2: 00$ am and maximum value $-5^{\circ} \mathrm{C}$ at 14:00 pm. Within the time window, temperature changes with time in the relationship of sine function. Precipitation changes by $20 \%$ randomly every five minutes on the basis of $1 \mathrm{~mm} / \mathrm{h}$.

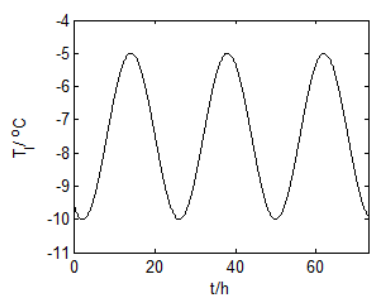

(a) temperature

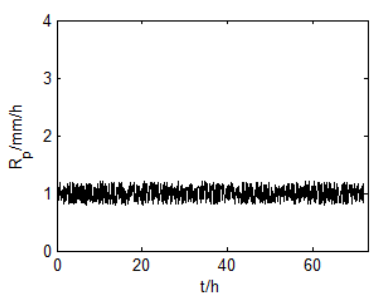

(b) precipitation

Fig.8 Ambient weather information of temperature and precipitation.

In order to reflect the regional climate differences, different wind speeds are set in different areas as listed in TABLE II.

TABLE II

WIND SPEEDS OF DIFFERENT AREAS

\begin{tabular}{cc}
\hline Parameter & Wind speed $/(\mathrm{m} / \mathrm{s})$ \\
\hline AREA 1 & $5 \pm 20 \%$ \\
AREA 2 & $8 \pm 20 \%$ \\
AREA 3 & $8 \pm 20 \%$ \\
\hline
\end{tabular}

\section{3) Simulation Settings}

Set the duration of the meteorological events as 72 hours with the step of 2 seconds and power system transient simulation as 20 seconds with the step of 0.01 seconds, respectively.

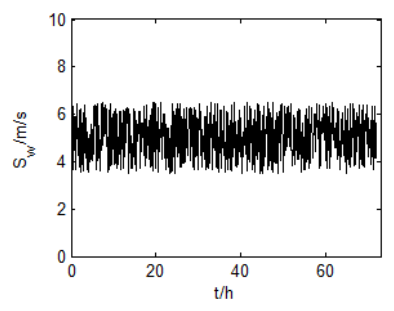

(a) Wind speed of AREA 1

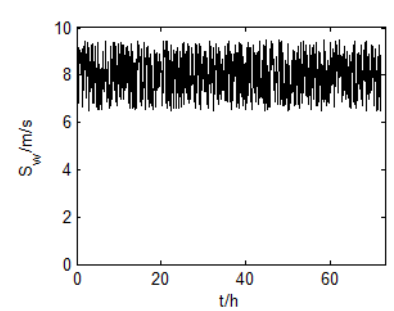

(b) AREA 2 and 3
Fig.9 Wind speeds of different areas.

Based on the 23-bus model of PSS/E, hybrid simulation is conducted with the input meteorological information. In the simulation, three processes are conducted. With the input of meteorological information, the process of ice accretion is simulated in Python. Electromechanical transient simulation is triggered when a mechanical event happens. Power flow calculation is performed following a load change. The last two processes are done with PSS/E. Then the simulation results are exported, e.g. lines breaking time, ice accretion growth rate and electrical variables like line current and active/reactive power, for further analysis.

\section{B. Simulation Results}

Simulation results are shown in Fig. 10 - Fig. 13. It can be seen from Fig. 10 that the ice accretion amount of eight transmission lines keeps rising during the simulation period. It can be observed that Lines \#5, \#6, \#2, \#1 and \#3 break after being exposed to the icing weather conditions 47.02, 54.20, 59.36, 60.00 and 60.30 hours, respectively. The ice accretion growth rate of line \#1 and \#5 are shown in Fig. 11.

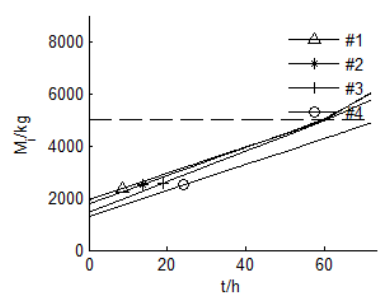

(a) $500 \mathrm{kV}$

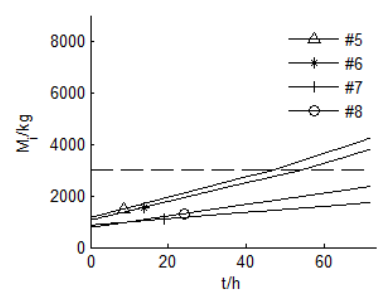

(b) $220 \mathrm{kV}$
Fig.10 Ice load on transmission lines.

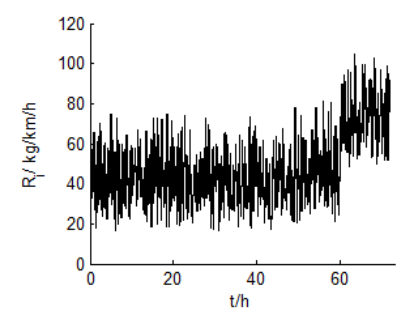

(a) line \#1

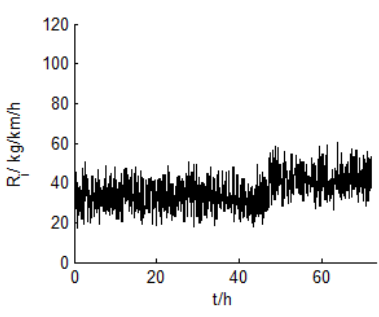

(b) line \#5
Fig.11 The time varying growth rate of ice accretion on different transmission lines.

It can be seen from Fig. 11 that the growth rates of ice accretion on line \#1 and \#5 have a jump increase at the corresponding line breaking time. When a line is tripped, the current of it will disappear, which helps increase the ice accretion growth rate. The current of the eight transmission lines during the simulation period is shown in Fig. 12. Security assessment shows that no currents of the lines hit the rating limits during the simulation period.

The effect of current change caused by line breaking on the ice accretion rate can be revealed by the hybrid simulation platform. Take transmission Line 1 as example, when it broke at hour 60.00, the current of it became zero and the ice accretion rate increased to a higher level. The dynamic interaction process of current and ice accretion rate is shown in Fig.13. It shows that line current has an effect on ice accretion, the interaction of icing weather events and power systems need to be modeled in a unified platform.

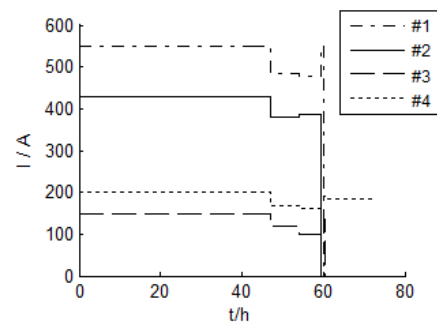

(a) $500 \mathrm{kV}$

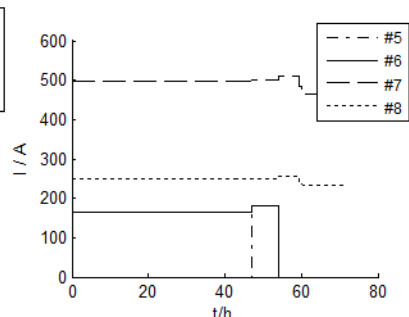

(b) $220 \mathrm{kV}$
Fig.12 Current magnitudes of transmission lines during the simulation period. 


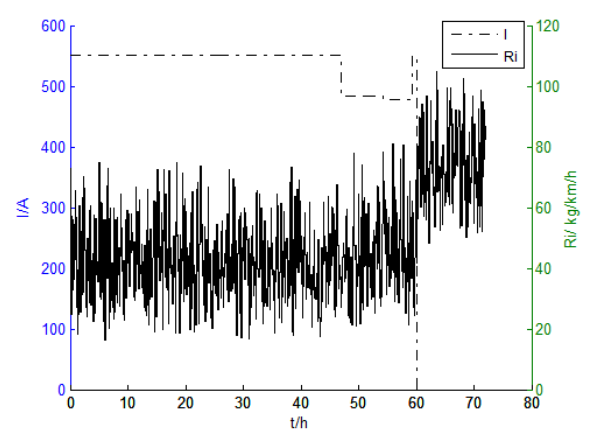

Fig.13 Current and ice accretion rate of transmission line \#1.

When line tripping occurs, two main changes will occur. Firstly, the generation is redispatched to meet the load. Secondly, multiple transmission lines tripping changes the system topology and the power flow is changed accordingly which leads to the current increasing or decreasing in other lines.

When line breaking occurs, the dynamic process of rotor angles can also be recorded as shown in Fig. 14. When line 6 breaks at hour 54.20, the angle differences between generators fluctuate and turn to be stable in twenty seconds.

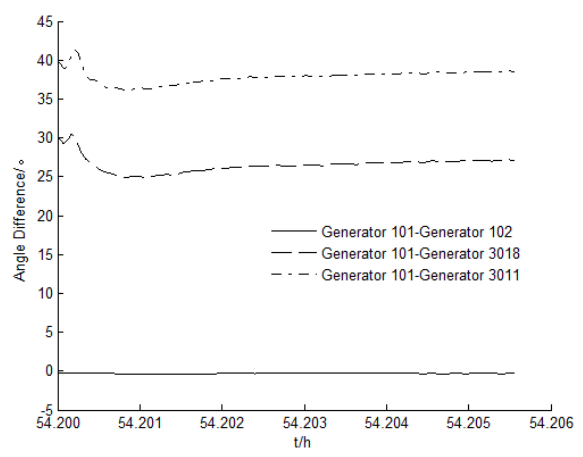

Fig.14 Angle differences between generators

As discussed above, ice accretion of transmission lines is affected by various factors, especially affected by ice accretion models adopted. Actually, ice accretion models are implemented by Python in the proposed platform, the simulation process based on any other ice accretion models can be easily implemented. It shows the flexibility of the proposed hybrid simulation scheme.

\section{Efficiency of Hybrid Simulation}

Twelve cases were tested to illustrate the efficiency of the proposed hybrid simulation approach, and the results are listed in TABLE III. They were all simulated in a desktop computer with four CPUs. The time used for the hybrid simulation is limited by the CPU clock speed.

$$
\text { TABLE III }
$$

Time Consuming List OF Hybrid Simulation Cases

\begin{tabular}{cccccccccc}
\hline $\begin{array}{c}\text { Simulation } \\
\text { period/hour }\end{array}$ & 24 & 24 & 24 & 48 & 48 & 48 & 72 & 72 & 72 \\
\hline $\begin{array}{c}\text { Number of } \\
\text { fault } \\
\text { time }\end{array}$ & 0 & 3 & 6 & 0 & 3 & 6 & 0 & 3 & 6 \\
consuming/sec & 6 & 10 & 16 & 12 & 17 & 22 & 19 & 23 & 28 \\
\hline
\end{tabular}

From the results in TABLE III, it can be observed that: a)The dynamic process of 72 hours can all be simulated within 28 seconds, and when there is no transmission lines breaking, the time will be shortened to 19 seconds; b)When the number of line breaking stays unchanged, the increase of 24 hours in the hybrid dynamic simulation will consume another 5 or 6 seconds; c)When the time period of hybrid dynamic simulation stay unchanged, additional several seconds will be consumed with the increase of transmission line breaking accidents.

The numerical approach for the hybrid simulation is very efficient according to the simulations results. The time used in the hybrid simulation can be divided into three parts: meteorological events simulation implemented with python, power flow calculation with load fluctuation and power system transients simulation based on PSS/E. For the first two, the time consumed mostly depends on the time period of simulation. For the third process, the number of disturbance (i.e. transmission line breaking) plays a crucial role.

\section{CONCLUSION}

A numerical approach for the hybrid simulation of power systems with extreme icing events is proposed. It integrates icing event with power system dynamics in a unified simulation framework, and reveals their interaction on each other. Two dynamic processes with quite different time scales are simulated with high efficiency, which provides operators an overview of the ice increasing and line switching process when experiencing extreme icing weather conditions.

An extended package for PSS/E enabling hybrid simulation of icing events and power system disturbances has been developed, based on which a hybrid simulation platform is established. Numerical studies show that the functionality of power system simulation is greatly extended by taking into account the icing weather events.

The hybrid simulation issue is a very challenging work, e.g. weather events modeling, the transformation from weather events to power system disturbances, and numerical simulation approaches in high efficiency are not well done in the literature. Researches on wind loading modeling, re-dispatch strategy, and power system risk assessment with extreme weather events will be carried out in the future work.

\section{REFERENCES}

[1] B. Ruszczak, and M. Tomaszewski, "Extreme Value Analysis of Wet Snow Loads on Power Lines," IEEE Trans. Power Syst., vol. 30, no. 1, pp. 457-462, Jan. 2015.

[2] G. Andersson, P. Donalek, R. Farmer, N. Hatziargyriou, I. Kamwa, P. Kundur, N. Martins, J. Paserba, P. Pourbeik, J. Sanchez-Gasca, R. Schulz, A. Stankovic, C. Taylor and V. Vittal, "Causes of the 2003 major grid blackouts in North America Europe, and recommended means to improve system dynamic performance,” IEEE Trans. Power Syst., vol. 20, no. 4, pp. 1922-1928, Nov. 2005.

[3] M. Hume, Environment Canada warns more bad weather coming to Metro Vancouver. [Online]. Available: http://www.theglobeandmail.com/news/british-columbia/windstorm-causes-hundred s-of-thousands-of-power-outages-in-southwest-bc/article26156 
$947 /$.

[4] A. Kenward, and U. Raja, "Blackout: Extreme Weather, Climate Change and Power Outages," Climate Central, pp. 1-23, 2014.

[5] P. Hines, J. Apt, and S. Talukdar, "Trends in the history of large blackouts in the United States," in Proc. 2008 IEEE Power \& Energy Society General Meeting, 2008.

[6] L. E. Kollar, M. Farzaneh, and P. Van Dyke, "Modeling ice shedding propagation on transmission lines with or without interphase spacers," IEEE Trans. Power Del., vol. 28, no. 1, pp. 261-267, Jan. 2013.

[7] E. Karimi, and A. Ebrahimi, "Inclusion of Blackouts Risk in Probabilistic Transmission Expansion Planning by a Multi-Objective Framework,” IEEE Trans. Power Syst., vol. 30, no. 5, pp. 2810-2817, Sept. 2015.

[8] N. Zhang, C. Kang, J. Liu, J. Xin, J. Wan, J. Hu and W. Wei, "Mid-short-term risk assessment of power systems considering impact of external environment," Journal of Modern Power Systems and Clean Energy, vol. 1, no. 2, pp. 118-126, Feb. 2013.

[9] X. Liu, M. Shahidehpour, Y. Cao, Z. Li and W. Tian, "Risk Assessment in Extreme Events Considering the Reliability of Protection Systems," IEEE Trans. Smart Grid, vol. 6, no. 2, pp. 1073-1081, Mar. 2015.

[10] X. Li, X. Zhang, L. Wu, P. Lu and S. Zhang, "Transmission Line Overload Risk Assessment for Power Systems With Wind and Load-Power Generation Correlation,” IEEE Trans. Smart Grid, vol. 6, no. 3, pp. 1233-1242, May 2015.

[11] M. J. Moser, T. Bretterklieber, H. Zangl and G. Brasseur, "Strong and Weak Electric Field Interfering: Capacitive Icing Detection and Capacitive Energy Harvesting on a 220-kV High-Voltage Overhead Power Line," IEEE Trans. Ind. Electron., vol. 58, no. 7, pp. 2597-2604, Jul. 2011.

[12] K. Savadjiev, and M. Farzaneh, "Modeling of icing and ice shedding on overhead power lines based on statistical analysis of meteorological data," IEEE Trans. Power Del., vol. 19, no. 2, pp. 715-721, Apr. 2004.

[13] W. Li, J. Zhou, and X. Xiong, "Fuzzy Models of Overhead Power Line Weather-Related Outages," IEEE Trans. Power Syst., vol. 23, no. 3, pp. 1529-1531, Aug. 2008.

[14] B. Yan, K. Chen, Y. Guo, M. Liang and Q. Yuan, "Numerical Simulation Study on Jump Height of Iced Transmission Lines After Ice Shedding," IEEE Trans. Power Del., vol. 28, no. 1, pp. 216-225, Jan. 2013.

[15] M. Farzaneh, and K. Savadjiev, "Statistical analysis of field data for precipitation icing accretion on overhead power lines," IEEE Trans. Power Del., vol. 20, no. 2, pp. 1080-1087, Apr. 2005.

[16] M. Huneault, C. Langheit, and J. Caron, “Combined models for glaze ice accretion and de-icing of current-carrying electrical conductors (J),” IEEE Trans. Power Del., vol. 20, no. 2, pt. 2, pp. 1611-1616, Apr. 2005.

[17] M. Huneault, C. Langheit, R. St.-Arnaud, J. Benny, J. Audet, and J. C. Richard, "A dynamic programming methodology to develop de-icing strategies during ice storms by channeling load currents in transmission networks," IEEE Trans. Power Del., vol. 20, no. 1, pt. 2, pp. 1604-1610, Apr. 2005.

[18] M. Farzaneh, and W. Chisholm, "50 years in icing performance of outdoor insulators," IEEE Electrical Insulation Magazine, vol. 30, no. 1, pp. 14-24, Feb. 2014.

[19] I. Imai, "Studies on ice accretion," Researches on Snow and Ice, vol. 3, no. 1, pp. 35-44, 1953.

[20] R. W. Lenhard, "An indirect method for estimating the weight of glaze on wires," Bulletio of American Meteorological Society, vol. 36, no. 3, pp. 1-5, 1955.

[21] E. J. Goodwin, J. D. Mozer, A. M. Digioia and B. A. Power, "Predicting ice and snow loads for transmission lines," in Proc. of the First International Workshop on Atwospheric Icing of Structures, pp. 267-273, Jun. 1983.

[22] L. Makkonen, "Modeling power line icing in freezing precipitation," Atmospheric Research, vol. 46, no. 1-2, pp. 131-142, Apr. 1998.

\section{BIOGRAPHIES}

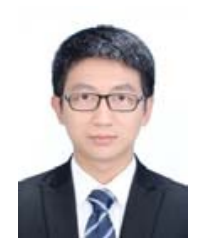

Lizheng Chen received his B.E. degree in electrical engineering from Shandong University in 2012. He is now a Ph.D candidate with the Key Laboratory of Power System Intelligent Dispatch and Control of Ministry of Education(Shandong University), P. R. China. His main research interests are power system numerical simulation, security and risk assessment.

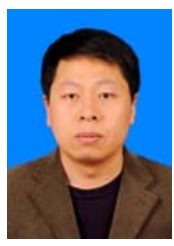

Hengxu Zhang(M'06)received his B.E. degree in electrical engineering from Shandong University of Technology, in 1998, and his M.S. and Ph.D. in electrical engineering from Shandong University, in 2000 and 2003, respectively. He is now a professor with the Key Laboratory of Power System Intelligent Dispatch and Control of Ministry of Education (Shandong University),P. R. China. His main research interests are power system security and stability assessment, power system monitoring and numerical simulation.

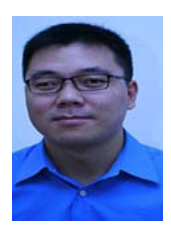

Qiuwei Wu (M'08-SM'15) obtained the B. Eng. and M. Eng. from Nanjing University of Science and Technology, Nanjing, P. R. China, in 2000 and 2003, respectively, both in Power System and Its Automation. He obtained the PhD degree from Nanyang Technological University, Singapore, in 2009, in Power System Engineering. He is an Associate Professor with the Department of Electrical Engineering, Technical University of Denmark (DTU). He is also a visiting professor named by Y. Xue, at Shandong University, China. His research interests are integration of wind power and electric vehicle into power systems, active distribution networks, and real time power system simulation and analysis. He is an Editor of IEEE Transactions on Smart Grid and IEEE Power Engineering Letters. He is also an Associate Editor of International Journal of Electrical Power and Energy Systems.

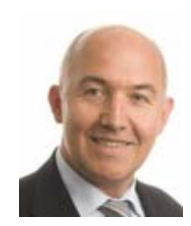

Vladimir Terzija (M'95-SM'00-F'16) was born in Donji Baraci (former Yugoslavia). He received the Dipl-Ing., M.Sc., and Ph.D. degrees in electrical engineering from the University of Belgrade, Belgrade, Serbia, in 1988, 1993, and 1997, respectively. He is the Engineering and Physical Science Research Council Chair Professor in Power System Engineering with the School of Electrical and Electronic Engineering, The University of Manchester, Manchester, U.K. His current research interests include smart grid application of intelligent methods to power system monitoring, control, and protection; wide-area monitoring, protection, and control; switchgear and fast transient processes; and digital signal processing applications in power systems. Prof. Terzija is Editor in Chief of the International Journal of Electrical Power and Energy Systems, Alexander von Humboldt Fellow, as well as a DAAD and Taishan Scholar. 